\title{
Espacio público y transporte masivo: usos y percepciones. Paseo Alcalde y línea 3 SITEUR en el Área Metropolitana de Guadalajara, México
}

\author{
María Teresa Pérez Bourzac ${ }^{1}$ | Carlos Alberto Crespo Sánchez ${ }^{2}$ | María Luisa García Yerena ${ }^{3}$ \\ Recibido: 21-07-2020 | Versión final: 04-02-2021
}

Resumen

\begin{abstract}
El interés investigativo que da origen al presente artículo se centra en analizar la relación entre las infraestructuras de movilidad y el espacio público generado para potencializar desplazamientos intermodales, relación que trasciende su sentido utilitario y se establece como un proceso de transformación social, económica y ambiental de entornos urbanos. El objetivo particular se centra en analizar los usos y percepciones que tiene lugar en el recién inaugurado espacio público Paseo Fray Antonio Alcalde, ubicado en el primer cuadro del Centro Histórico de la ciudad de Guadalajara, México. Espacio transformado que presenta nuevas cualidades socio-espaciales derivadas de su estrecho vínculo con la construcción de la línea 3 del Tren Eléctrico Urbano (TEU), infraestructura de movilidad de impacto metropolitano. Es inherente a toda propuesta que conlleve la transformación de un centro histórico el diálogo entre posturas antagonistas, entre quienes abogan por recobrar esencias y quienes vislumbran futuros progresistas con nuevos códigos, en medio de ellos la población urbana que usa, vive y transforma dichos espacios a partir de sus prácticas cotidianas, a ellos se les da voz en el presente abordaje. La metodología empleada vinculó el análisis histórico con un ejercicio de análisis perceptual a partir de entrevistas semiestructuradas y observación no participante en el área de estudio. Derivado de ello, la percepción predominante etiqueta al proyecto como "terreno ganado", remitiéndose a los recuerdos aún vívidos de representaciones pasadas, no obstante, estableciendo visiones críticas de lo existente y exigencias claras de hacer valer el interés común sobre intereses económicos particulares.
\end{abstract}

Palabras clave: Apropiación social; transporte público; centro histórico

Citación

\section{Public Space and Mass Transportation: Uses and Perceptions. Paseo Alcalde and Line 3 SITEUR in the Metropolitan Area of Guadalajara, México}

Abstract

\begin{abstract}
The research interest that gives rise to this article centers on analyzing the relationship between mobility infrastructures and the public space generated to potentiate intermodal movements, a relationship that transcends its utilitarian sense and is established as a process of social, economic and environmental transformation of urban environments. The particular objective is focused on analyzing the uses and perceptions that take place on the recently inaugurated public space "Paseo Fray Antonio Alcalde" located in the first square of the Historic Center of the city of Guadalajara, Mexico. Transformed space that presents new socio-spatial qualities derived from its close link with the construction of line 3 of the Urban Electric Train System (SITEUR), a metropolitan impact mobility infrastructure. Inherent in any proposal that involves the transformation of a historic center is the dialogue between antagonistic positions, between those who advocate recovering Essences and those who envision future progressives with new codes, among them the urban population that uses, lives and transforms said spaces from their daily practices, they are given a voice in the present approach. The methodology used linked the historical analysis with a perceptual analysis exercise based on semi-structured interviews and non-participant observation in the study area. Derived from this, the predominant perception labels the project as "gained ground", referring to the still vivid memories of past representations, however, establishing critical visions of the existence and clear demands to assert the common interest over private interests.
\end{abstract}

Keywords: Social appropriation; public transport; historic centre

${ }^{1}$ Doctora en Historia del Arte por la Universidad de Las Palmas de Gran Canarias, España. Investigadora del Instituto de Investigación y Estudios de la Ciudad, Universidad de Guadalajara (ORCiD: 0000-0002-0851-7982), ${ }^{2}$ Doctor en Ciudad, Territorio y Sustentabilidad por la Universidad de Guadalajara. Investigador del Instituto de Investigación y Estudios de la Ciudad. Universidad de Guadalajara (ORCiD: 0000-0001-8312-9368; Scopus autor ID: 57212269750), ${ }^{3}$ Doctora en Arquitectura por la Universidad de Las Palmas de Gran Canarias, España. Investigadora del Instituto de Investigación y Estudios de la Ciudad. Universidad de Guadalajara (ORCiD: 0000-0001-7675-4536). Correos de contacto: mayte.pbourzac@academicos.udg.mx; carlos.crespo@academicos.udg.mx; maria.gyerena@academicos.udg.mx. 


\section{Introducción}

El presente trabajo tiene como objetivo el análisis de los usos y percepciones que se suscitan en el nuevo espacio público Paseo Fray Antonio Alcalde (abril 2020) ubicado en el primer cuadro del Centro Histórico de la ciudad de Guadalajara, México. Espacio transformado que presenta nuevas cualidades socioe-spaciales derivadas de su estrecho vínculo con la construcción de la línea 3 del Sistema del Tren Eléctrico Urbano (SITEUR), infraestructura de movilidad iniciada en el año 2014 e inaugurada en mayo del 2020, cuyo impacto e influencia se extiende a la escala Metropolitana. El análisis de esta relación requiere en principio de un abordaje teórico sobre los procesos de percepción en el espacio público, en el contexto de los megaproyectos como lo son las infraestructuras de movilidad para el transporte masivo; y posteriormente de un acercamiento de carácter histórico, cualitativo-descriptivo del entorno consolidado del primer cuadro del centro de la ciudad que aloja a este nuevo espacio público y su vínculo con la infraestructura de movilidad. El enfoque elegido permite establecer antecedentes y propiciar reflexiones derivadas de los principales resultados de una encuesta de percepción y evaluación de este nuevo espacio realizada a visitantes de la zona durante el verano del 2019. Las conclusiones mostrarán el grado de transformación social de un espacio público en un contexto histórico a partir de un megaproyecto materializado en la construcción de una infraestructura de movilidad.

El proyecto Paseo Fray Antonio Alcalde consiste en una intervención urbana conocida como calle compartida, espacio donde coinciden ciclistas, peatones y tráfico local, y sus espacios están delimitados por la señalética, mobiliario urbano y vegetación. Su longitud se extiende a lo largo de 2,5 $\mathrm{km}$ en un sentido norte - sur, contactando en sus dos extremos la glorieta conocida como "La Normal" hasta los templos de Aranzazú y San Francisco en el centro de Guadalajara; todo ello logrado con una intensa reforestación y sustitución de mobiliario urbano, instalaciones eléctricas, sanitarias y pluviales. Luego de adelantados los trabajos de la Línea 3 del Sistema de Tren Eléctrico Urbano (SITEUR), el paseo se ha extendido hacia el sur en la zona conocida como de los dos templos (San Francisco y Aranzazú), hasta la Calzada Independencia, actualmente ya está terminado.

El interés del estudio está centrado en el análisis de la relación entre la infraestructura de movilidad y el espacio público producido, desde una óptica de percepción socio-espacial. Lo anterior proporcionó algunos fundamentos para la definición de las siguientes preguntas orientadoras: ¿Cuáles cambios se observan en el uso y apropiación del espacio público con la implementación de esta infraestructura de movilidad? ¿cuál ha sido la percepción de los visitantes respecto a la implementación del Paseo? ¿los usos y funciones de este espacio público han evidenciado cambios por la construcción de esta infraestructura?

El trabajo aquí expuesto se deriva del desarrollo de un proyecto de investigación de mayor alcance y proyección que se desarrolla bajo el título "Transformaciones de carácter social, ambiental y territorial, resultado del desarrollo de proyecto de movilidad. Estudios de caso: Sistema Integrado de Transporte (Municipio de Bello) Bogotá y su relación futura con el Tren de cercanías; Tren interurbano del Valle de Toluca, mediante un estudio paralelo de la Línea 3 del Sistema de Tren Urbano (SITEUR) del Área Metropolitana de Guadalajara, Jalisco". Este adelanto investigativo se expone a la evaluación del lector y su retroalimentación.

\section{Usos y percepciones sociales de la ciudad}

La percepción se establece como detonante del comportamiento individual y colectivo al interactuar con un medio. Según lo describe Downs (1970) ésta se formula a partir de un proceso complejo en el que receptores de percepción abstraen información del medio a partir del estímulo de sentidos

ACE, 16 (47) CC BY-ND 3.0 ES | UPC Barcelona, España | Espacio público y transporte masivo: usos y percepciones.

Paseo Alcalde y línea 3 SITEUR en el Área Metropolitana de Guadalajara, México. DOI http://dx.doi.org/10.5821/ace.16.47.9631 
corporales, configuradores esenciales de la experiencia. La información se filtra por medio de un sistema de valores construido a partir experiencias pasadas, directas o indirectas, ideales preconcebidos, estereotipos, estigmas, que se encuentran alojados en la memoria, este sistema de valores a su vez formula una imagen del entorno que funge como pieza resultante de procesos de evaluación conscientes o inconscientes. Esta imagen construida determina la decisión y el comportamiento del sujeto o los colectivos que actúan directamente sobre el medio real estableciendo usos, prácticas y actividades. Es así como el medio percibido y el medio real se retroalimentan constantemente fundiéndose en un proceso recursivo permanente. Las percepciones, individuales y colectivas, son pieza clave dentro del proceso de construcción de lugares y con ello, del proceso mismo de conformación de una sociedad urbana. Tal como lo argumenta Park "indirectamente y sin tener plena conciencia de la naturaleza de su obra, al crear la ciudad, el hombre se recrea a sí mismo” (1999, pág. 155)

¿Por qué es importante el estudio de las percepciones y los usos sociales de la ciudad? La ciudad se caracteriza por ser la superficie dispuesta para el desarrollo de usos, prácticas y actividades sociales diversas, esta se configura a partir de la disposición de elementos materiales, lo que Lefevbre (1974) llamaría el espacio concebido, los cuales fungen como plataformas de soporte para sus atributos subjetivos que le dotan de dinamismo y se amparan en el tiempo, el espacio percibido y espacio vivido, en palabras del mismo Lefebvre. Tanto los soportes como las prácticas que se ejecutan en ellos funcionan bajo una relación de dependencia mutua. El espacio público, principal articulador de la vida urbana, depende directamente de los procesos colectivos de apropiación, de ser asimilado como plataforma dispuesta para el ejercicio político constante y permanente entre la heterogeneidad social que conforma a los contextos urbanos, sólo a partir de que logra ser asimilado de esta forma se puede hablar de su consolidación. Por tanto, cada nuevo soporte propuesto en la ciudad existe y permanece solo a partir de que pasa por procesos perceptuales de asimilación y dotación de usos colectivos. El logro del objetivo para el cual estos soportes son pensados, diseñados y puestos a disposición se encuentra condicionado por las valoraciones subjetivas e intersubjetivas que emanen de la experiencia urbana cotidiana.

\section{Las infraestructuras de movilidad y el transporte masivo en la ciudad contemporánea}

Las ciudades protagonizan cambios y transformaciones relevantes como resultado de intervenciones de gran escala y la implantación de lo que se denomina grandes proyectos o megaproyectos (Díaz Orueta, 2009). Existe una amplia contribución teórica sobre este tipo de intervenciones y en ese ámbito de estudio, Díaz Orueta (2009) aporta el término megaproyecto urbano e impulsa investigaciones sistemáticas y críticas sobre el impacto de los megaproyectos urbanos en las grandes ciudades y transmitiendo una preocupación colectiva por las consecuencias sociales y ambientales que acarrea este modelo de intervención territorial. El megaproyecto urbano se refiere a dos tipos de intervención urbana: una, relativa a obras arquitectónicas que dotan de carga simbólica a zonas relegadas de la ciudad; y la otra, inherente a proyectos más complejos que integran áreas residenciales, comerciales e infraestructura pública, que establecen la colaboración entre el sector público y privado.

Los megaproyectos se convierten en uno de los ejes fundamentales de políticas de reestructuración urbana. Tendencia reiterada que puede observarse en diversas ciudades y cuyo impacto no se restringe a áreas urbanas concretas, sino que influye al conjunto de la escala metropolitana y con ello el surgimiento de nuevos patrones de estructuración socio-territorial que responden procesos de globalización económica y que las ciudades tienen que asumir y adaptar (Díaz Orueta, 2015). En general, y a diferencia de los proyectos tradicionales, un megaproyecto o un gran proyecto de

ACE, 16 (4.7) CC BY-ND 3.0 ES | UPC Barcelona, España | Espacio público y transporte masivo: usos y percepciones.

Paseo Alcalde y línea 3 SITEUR en el Área Metropolitana de Guadalajara, México. DOI http://dx.doi.org/10.5821/ace.16.47.9631 
infraestructura tiene un tiempo de vida mucho mayor, en virtud de que son infraestructuras de gran impacto que pueden tener 50 o 100 años de vigencia en el futuro, como es el caso de las infraestructuras de movilidad y de transporte. La determinación del valor de beneficios, costos, entre otros, se convierte en un reto, especialmente en contextos como el mexicano, donde hay procesos institucionales complejos y limitaciones a la información. Por tanto, es necesario la realización de estudios específicos y la existencia de observatorios que permitan analizar el comportamiento de los proyectos de mediano y largo plazo, lo que generará información con un valor público apreciado, como una condición necesaria que hará posible la transferencia de la experticia técnica y la transferencia de recursos y conocimientos en este ámbito (Roa, 2010). Es pertinente mencionar que los megaproyectos han venido a dar un nuevo enfoque a la organización territorial, nuevas relaciones económicas, políticas y sociales, interés por las grandes inversiones desde organismos públicos y privados (Pérez-López, 2019). Lo que genera un nuevo espacio de oportunidad para analizar desde sus dimensiones materiales y simbólicas (Talledos e Ibarra 2016).

Los megaproyectos de infraestructuras de movilidad en las ciudades contemporáneas se han convertido en un asunto de interés público e importancia por los impactos que generan en la estructura urbana y territorial.

Las infraestructuras de transporte son elementos clave en la estructura espacial del territorio, por las relaciones que facilitan entre diferentes componentes del mismo. La intensidad de dichas relaciones define las características de conectividad, accesibilidad, movilidad y facilita la dispersión y la descentralización de las actividades urbanas. (Suárez Pradilla \& Roca Cladera, 2019)

Las ciudades del contexto latinoamericano también intentan ocuparse de los problemas de movilidad acuciantes buscando reducir el aforo vehicular particular e impulsar proyectos de transporte público en sus entornos metropolitanos.

Las perspectivas en el estudio del transporte público se diversifican, pero para el desarrollo de este trabajo se consideró oportuno elegir a autores cómo Carrillo, Córdova, Arellano, Calonge, MirallesGuasch y Cebollada, que aportan algunas ideas para el establecimiento de un marco teórico. En esta tesitura es en la década de 1990 que Salvador Carrillo (1994) en sus estudios había identificado en América Latina cinco perspectivas en el estudio del transporte público. La primera eran las investigaciones centradas en la demanda; la segunda en los estudios sectoriales; la tercera la que se inscribía en la planificación urbana; en un cuarto ramal, la centrada en la oferta; y, finalmente, las de tipo sociológico, que analizaba la relación Estado, transporte y usuarios. Posteriormente Arellano Ríos (2018) aporta una interesante clasificación sobre estudios del transporte público y perspectivas que estructura en cuatro enfoques: las que van desde las perspectivas técnico-planificadoras, las geoeconómicas, las socio-urbanas, hasta algunas más actuales de difusión que incluyen la participación de la sociedad civil (Arellano Ríos, 2018). Esta última perspectiva es de crucial interés porque hace énfasis en rescatar el espacio público (Romero Pérez, 2011). Dichas perspectivas, además, adoptan algunas directrices de paradigmas actuales como el cambio climático, la movilidad urbana sustentable y desarrollo humano.

Es importante, para poder definir la sostenibilidad de una infraestructura de transporte, conocer los costes resultantes y el nivel de accesibilidad, según plantea Miralles-Guasch y Cebollada (2003): es imprescindible, para valorar la eficiencia y por lo tanto el coste de los medios de transporte, que se incluyan variables como el consumo de recursos naturales no renovables, el volumen de contaminantes emitidos a la atmósfera, al agua y al suelo, las garantías de seguridad para el conjunto de la población, el nivel de convivencia entre los distintos medios de transporte, la extensión del suelo ocupado por las infraestructuras y los medios de transporte, la distribución de los usos de la calle en relación al transporte y al resto de actividades socio-económicas, así como el precio que se debe pagar colectivamente por el mantenimiento del modelo de movilidad (pág. 13).

ACE, 16 (47) CC BY-ND 3.0 ES | UPC Barcelona, España | Espacio público y transporte masivo: usos y percepciones.

Paseo Alcalde y línea 3 SITEUR en el Área Metropolitana de Guadalajara, México. DOI http://dx.doi.org/10.5821/ace.16.47.9631 
La ciudad de Guadalajara, México y su área metropolitana, se suman a la apuesta de invertir en infraestructuras de movilidad y sitúan al transporte público masivo como un eje prioritario en su desarrollo, desde el 2006, se impulsa el paradigma de la Movilidad Urbana Sustentable (Córdova España, 2010). Por un lado, se inicia la construcción de una nueva red de transporte público masivo con autobuses en carriles exclusivos, BRT (Bus de Tránsito Rápido, por sus siglas en inglés) y por otro lado en 2013 se aprueba la Ley de Movilidad considerada como una ley pragmática y de avanzada en el complejo sistema del transporte público. La ley establece seis ejes prioritarios: relación de la ley y políticas públicas, infraestructura, aspectos tecnológicos del servicio, semaforización, desplazamientos de la gente (observatorio ciudadano y un Instituto de Movilidad), imagen urbana e intermodalidad.

El transporte público masivo en Guadalajara acumula una historia de rezago por más de cuarenta años y tiene su primer antecedente en 1970, cuando se inicia la construcción de la primera línea de tren eléctrico en vez de metro, con un proceso de ajuste dilatado que finalmente concreta su puesta en marcha en 1989 como transporte masivo. En 1992 se vislumbra el inicio de las obras de la segunda línea de tren eléctrico, veinte años después se gesta la posibilidad del proyecto de la línea 3 del tren ligero, que ahora sí considera las características del transporte masivo del metro.

La línea 3 del SITEUR de Guadalajara, forma parte de una serie de intervenciones que se han desarrollado en la ciudad en los últimos años, que responden al deseo de legitimación que los gobernantes necesitan para evidenciar sus políticas para hacer de Guadalajara una ciudad sustentable, con todos los significados que tiene esta palabra y que no forman parte de nuestros objetivos en esta ocasión. La construcción de la Línea 3 del SITEUR se inicia desde agosto de 2014, y con ello, el proyecto alberga la posibilidad de conectar los municipios metropolitanos de Zapopan, Guadalajara, Tlaquepaque y Tonalá, con el objetivo entre otros, de lograr la salida de circulación de 10,000 vehículos por jornada y eliminar la emisión de 17, 400 toneladas de dióxido de carbono a la atmósfera y de esta forma cumplir con acciones de mitigación del cambio climático (Gobierno del Estado de Jalisco, 2018). Tiene una longitud de $22 \mathrm{~km}$ y dispone de 18 estaciones, 13 de ellas elevadas y el resto subterráneas, proyectado para iniciar actividades en el 2016, pero que finalmente se inauguró el 12 de septiembre del 2020 (Figura 1).

Figura 1. Transporte público masivo en el Área Metropolitana de Guadalajara

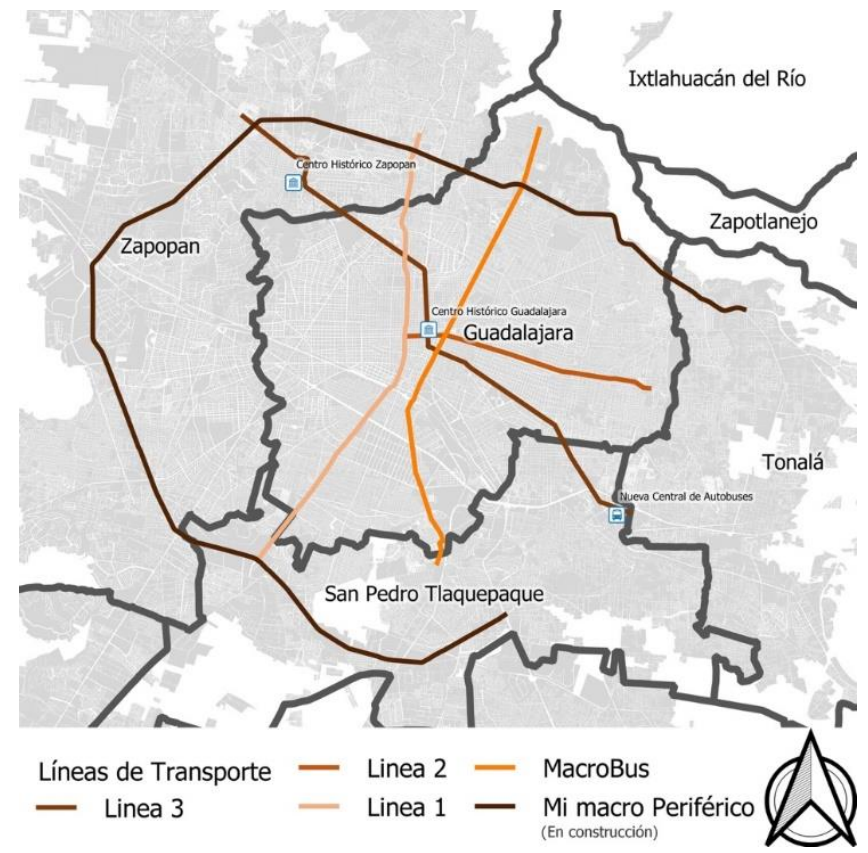

Fuente: Elaboración por parte de colaborador Yoav Alejandro Jiménez Aguas

ACE, 16 (47) CC BY-ND 3.0 ES | UPC Barcelona, España | Espacio público y transporte masivo: usos y percepciones.

Paseo Alcalde y línea 3 SITEUR en el Área Metropolitana de Guadalajara, México. DoI: http://dx.doi.org/10.5821/ace.16.47.9631 
Según el investigador Fernando Calonge (2017): Para garantizar que el levantamiento de una infraestructura de transporte masivo pueda incidir en una disminución de los desplazamientos en automóvil, en el aprovechamiento de los recursos y redes urbanos existentes, en el fomento de las movilidades activas y no motorizadas, o en su propia sustentabilidad operativa es preciso que venga acompañado de un número considerable de medidas complementarias... En este sentido, mejorar la combinación de usos de suelo, las condiciones de caminabilidad, el entorno urbano, alentar medidas que ayuden a redensificar los espacios, son intervenciones que habría que observar que acompañan a la construcción de una infraestructura como la Línea 3 del Tren Ligero para garantizar mayores cuotas de sustentabilidad (pág. 22)

De acuerdo con datos proporcionados por la Secretaría de Desarrollo Agrario, Territorial y Urbano (SEDATU) la aplicación de una estrategia de Desarrollo Orientado al Transporte (DOT) en el Área Metropolitana de Guadalajara implicaría la creación de barrios densamente poblados alrededor de las infraestructuras de transporte público masivo. Esta estrategia responde al crecimiento poblacional que no corresponde con la capacidad del transporte público existente, la pérdida de densidad habitacional, la expansión urbana incontrolada como incentivo al uso del transporte privado, y, se podría incentivar con ello, el uso de espacios subutilizados como viviendas vacías.

Con la consolidación de la Línea 3 del SITEUR, pero desde perspectivas optimistas, se ha asumido el reto de emprender acciones para transformar la movilidad, en factor de cambio en las ciudades, como una estrategia para generar condiciones de prosperidad urbana en el Área Metropolita de Guadalajara.

\section{Desarrollo histórico del espacio público de la zona de estudio (cruz de plazas), el centro y sus transformaciones}

La ciudad de Guadalajara, a partir de los procesos de modernización a los que la ciudad fue sometida en la segunda mitad del siglo XX, experimentó un proceso de expansión territorial que, aún hoy, no tiene límites y ha desarrollado un fenómeno de conurbación cada vez mayor. Ante este fenómeno de crecimiento expansivo y de transformación interior que comenzó por los años cincuenta del siglo XX, la ciudad transformó algunos espacios de referencia, emblemáticos, con grandes intervenciones que la modernizaron, entre otros ejemplos, la apertura de varias avenidas (16 de septiembre, Juárez y Federalismo) y el proyecto de la Cruz de Plazas (1948).

La imagen histórica del centro de la ciudad se fue consolidando alrededor de la Catedral de Guadalajara (1751) hito de referencia de la urbe, que destaca por su enorme valor Patrimonial Cultural y su arquitectura de gran valor, alrededor de la cual se estructuró el proceso de transformación espacial que supuso este proyecto, a la par que rediseñó el corazón mismo de la ciudad, añadiendo nuevos espacios y resignificando otros dentro del nuevo patrón y dinámica urbanas.

La plaza de Armas o Plaza Mayor, único espacio que se remonta a la última y definitiva fundación de la ciudad en 1542, representó durante muchos años, el espacio público de mayor referencia, espacio multifuncional que respondió a los poderes históricos que el paso de los años consolidó; el único espacio público jerárquicamente importante que unos años después, se convirtió en un elemento accesorio del nuevo proyecto urbano de la Cruz de Plazas. Guadalajara modifica así su estructura física, aunque pudo conservar las características de usos de sus espacios públicos más centrales a pesar de haber perdido elementos representativos que pudieran haberla significado, sin prejuicio sustantivo de la trama o tejido de su entorno inmediato y a pesar del expolio que supuso su construcción, por conservar los rasgos más representativos de la ciudad y sus dinámicas urbanas.

ACE, 16 (47) CC BY-ND 3.0 ES | UPC Barcelona, España | Espacio público y transporte masivo: usos y percepciones.

Paseo Alcalde y línea 3 SITEUR en el Área Metropolitana de Guadalajara, México. DOI http://dx.doi.org/10.5821/ace.16.47.9631 
Estas transformaciones y la posterior construcción del Conjunto Centro Metropolitano (Plaza Tapatía, 1977), donde fueron demolidas nueve manzanas y 15,000 m2 de calles, dio como resultado una transformación de 600 m, con 70 nuevos edificios (150 00 m2 de construcción), dos plazas y tres paseos y varios conjuntos escultóricos, además del proyecto integral de vialidad (túnel vehicular de Hidalgo, con tres estacionamientos), evidenciaron las transformaciones a que fue sometida la zona central de la ciudad, en un afán principal de rentabilizar el suelo público, dando como resultado la transformación de un espacio público urbano monumental, que en sus dimensiones, supuso la destrucción/transformación de la estructura, trama y tejido central de la ciudad, así como la pérdida de gran parte de su patrimonio arquitectónico singular y la desarticulación de toda la zona inmediata.

Ahora bien, la llegada de la modernidad a la ciudad dio paso a un cambio paulatino en los usos del suelo en la zona, lo que a su vez fue segmentando barrios tradicionales y sectores urbanos homogéneos, ricos en tradiciones culturales, en edificaciones y en espacios de gran valor patrimonial. La actividad comercial se siguió consolidando en el centro histórico e hizo de la terciarización en el uso de suelo un estandarte de la ocupación del espacio, con la consecuente baja del uso habitacional o el abandono de espacios por la población residente. Las transformaciones urbanas y los proyectos de acupuntura urbana en los espacios patrimoniales no son ajenos para la ciudad que busca ser competitiva, pero que no atina aún, con proyectos a largo plazo, la reactivación integral en el Centro Histórico.

\section{Proyecto del Paseo Fray Antonio Alcalde}

El proyecto del Paseo Alcalde se presenta y difunde como una intervención trascendental para el Área Metropolitana de Guadalajara, realizada en una de las áreas más importantes del Centro Histórico, que está logrando transformar de manera cualitativa el espacio público de la calle, cuyo cambio se hace evidente al pasar del uso exclusivo de la avenida para la movilidad de vehículos a una propuesta de mayor calidad espacial en su diseño de calle compartida y la definición de un espacio público para el uso y disfrute del peatón. A continuación, se describirán algunos antecedentes y las consideraciones del proyecto de dicho paseo.

\subsection{Antecedentes}

La Avenida Fray Antonio Alcalde - 16 de septiembre, en el tramo que comprende entre la calle Miguel Blanco y La Glorieta de la Normal, era una de las avenida de alta congestión vehicular y por donde convergían alrededor de cien rutas de transporte público, una carga vehicular significativa, que contaba con dos carriles en ambos sentidos, en ella circulaban más de 100 mil automóviles cada día y 45 rutas de transporte público cruzaban la ciudad utilizando esta avenida, con una frecuencia de paso promedio de 10 minutos, la más alta en la ciudad. Esto derivó en problemas de contaminación atmosférica, visual y auditiva, sobre todo. La avenida alcanzaba los 80 decibeles durante la actividad diurna, superando por mucho el nivel máximo de decibeles establecido en la NOM-081-SEMARNAT, de 68 decibeles (Instituto de Información Territorial, 2013).

La propuesta de conectividad de un medio de transporte masivo como la línea 3 del SITEUR, atravesando el corazón del Centro Histórico de Guadalajara, con una parte de forma elevada y en otra de forma subterránea, dio paso a la posibilidad de la construcción de un espacio público lineal que aprovechará la superficie y proporcionará una nueva fisonomía del entorno y en el patrimonio cultural. De esta inercia transformadora surge el proyecto "Paseo Fray Antonio Alcalde”, cuyos nuevos espacios ya están siendo utilizados de forma total desde el mes de abril del 2020.

ACE, 16 (47) CC BY-ND 3.0 ES | UPC Barcelona, España | Espacio público y transporte masivo: usos y percepciones.

Paseo Alcalde y línea 3 SITEUR en el Área Metropolitana de Guadalajara, México. DOI http://dx.doi.org/10.5821/ace.16.47.9631 
El Gobierno del Estado de Jalisco y el Municipio de Guadalajara crearon y gestionaron diversos instrumentos de carácter normativo para efectuar dicha intervención urbana. Instrumentos de planeación urbana como el Polígono de Intervención Especial (PIUE) en el año 2016 y el consecutivo Plan Rector, se presentan como una nueva herramienta para ordenar el espacio urbano consolidado que necesita de estrategias o proyectos urbanos para su reactivación urbana, social y económica. El Plan Rector del Polígono de Intervención Urbana Especial número seis, correspondiente al área de estudio denominada Corredor Alcalde - Centro Histórico y que a su vez se encontraba enmarcado en los ejes estratégicos del Plan y Programa Municipal de Desarrollo Urbano, que establece los ejes estratégicos y las condiciones para su instrumentación jurídica y para implementar iniciativas de regeneración urbana, reactivación de espacios urbanos subutilizadas, promover desarrollos orientados al transporte (DOT).

Posteriormente se publicó el Plan Maestro Centro Guadalajara (2017) que se presenta como un instrumento flexible, modificable, ampliable, evolutivo y con ello se establece la base para iniciar un mecanismo de gestión institucional, participativa e incluyente. Se pretenden acciones como: la renovación y conservación de espacios públicos; proyectos habitacionales de adaptación controlada en edificaciones patrimoniales en condición de subutilización o en estado ruinoso; premio y descuento en impuestos a la conservación de fincas patrimoniales; y el mejoramiento, reutilización y promoción de vivienda en edificios verticales existentes subutilizados no patrimoniales.

\subsection{Proyecto}

Ahora bien, el Paseo Fray Antonio Alcalde, como una obra de intervención urbana que tiene como objetivo principal reorganizar la estructura de la avenida a partir de su rediseño, convirtiéndola de una avenida de 4 carriles en ambos sentidos a un espacio diseñado bajo el concepto de "calle compartida" en la que conviven amablemente peatones, ciclistas y tránsito local, es el espacio que con estas nuevas características espaciales, contempla un proceso de reconversión a un espacio caminable que considera como usuario principal al peatón.

El proyecto tiene una longitud total de 2.5 kilómetros, en dirección norte-sur, conecta la glorieta de La Normal con el Jardín San Francisco, mejor conocido como los "dos templos" San Francisco y Aranzazú que, a su vez, conecta con recintos educativos, institucionales, patrimoniales, plazas y jardines, por su extensión es considerado el paseo lineal más extenso de México (Figura 2).

Figura 2. Esquema del Paseo Fray Antonio Alcalde

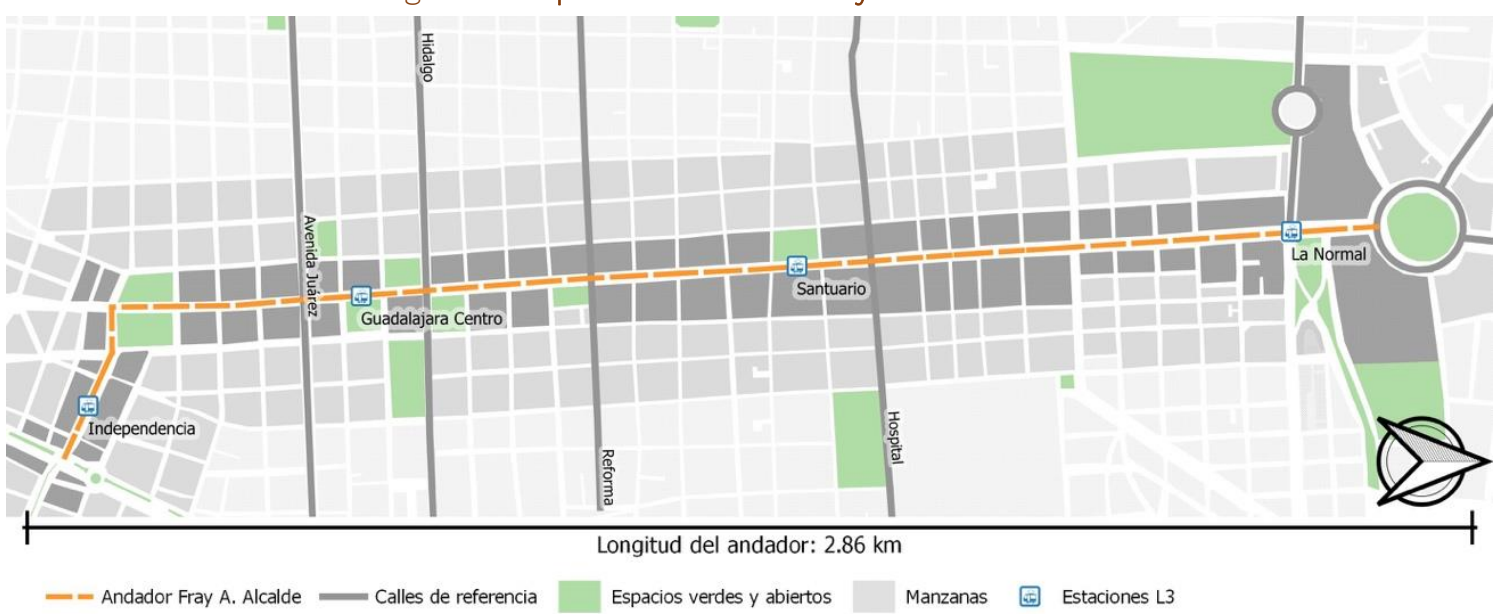

Fuente: Elaboración por parte de colaborador Yoav Alejandro Jiménez Aguas

ACE, 16 (4.7) CC BY-ND 3.0 ES | UPC Barcelona, España | Espacio público y transporte masivo: usos y percepciones. Paseo Alcalde y línea 3 SITEUR en el Área Metropolitana de Guadalajara, México. DOI: http://dx.doi.org/10.5821/ace.16.47.9631 
El entorno arquitectónico-urbano se cualifica con nuevos espacios de interacción, el cambio en su fisonomía y su relación con la arquitectura es notoria a partir de la transformación de la avenida Alcalde. El paisaje establece una nueva dialéctica con el patrimonio histórico, se llevan a cabo algunas modificaciones y remozamiento de fachadas. De acuerdo con el inventario patrimonial emitido en el Plan Maestro (Gobierno de Guadalajara, 2017) existen en el Paseo, edificios catalogados como Monumentos Históricos por Determinación de Ley, entre ellos se encuentran los templos de San Francisco y Aranzazú, San José, la catedral de Guadalajara, la Rotonda de los Jaliscienses Ilustres y el templo católico Santuario de Nuestra Señora de Guadalupe, con su plaza delante; esta catalogación considera la arquitectura realizada entre los siglos XVI y XIX destinados al uso público.

También conviven en su trayecto: Monumentos Históricos civiles relevantes por Determinación de Ley, arquitectura civil relevante realizada entre los siglos XVI y XIX; e Inmuebles de Valor Histórico Ambiental; denominación que incluye a edificios construidos antes de 1900, e inmuebles catalogados como de Valor Artístico Ambiental, referidas a edificaciones construidas después de 1900 que poseen valor contextual o de ambiente urbano generados en una zona de valor patrimonial. El valor patrimonial de las edificaciones que acompañan al trayecto le dotan de un valor histórico único.

En la parte norte del Paseo, aún se encuentran vestigios de la actividad de los barrios tradiciones de El Retiro al oriente, como sede del proyecto de la Ciudad Creativa Digital y El Santuario al poniente, barrio con carácter predominante de clase media, aunque con algunas casas señoriales, conocido por sus restaurantes/fondas de comida típica mexicana, cuyos entornos se han visto afectados por las nuevas dinámicas que están surgiendo en dicho espacio público.

El espacio público resultante de dicha intervención y que se identifica con grandes banquetas, mobiliario urbano adecuado para el descanso, fuentes, entre otros atributos espaciales está generando procesos de usos interesantes, ya se puede observar algunos procesos de apropiación durante la mañana y la tarde. La dinámica del uso del mobiliario urbano se hace evidente de forma intermitente por las condiciones climáticas y la vegetación que provee, de momento, de poca sombra (Figura 3).

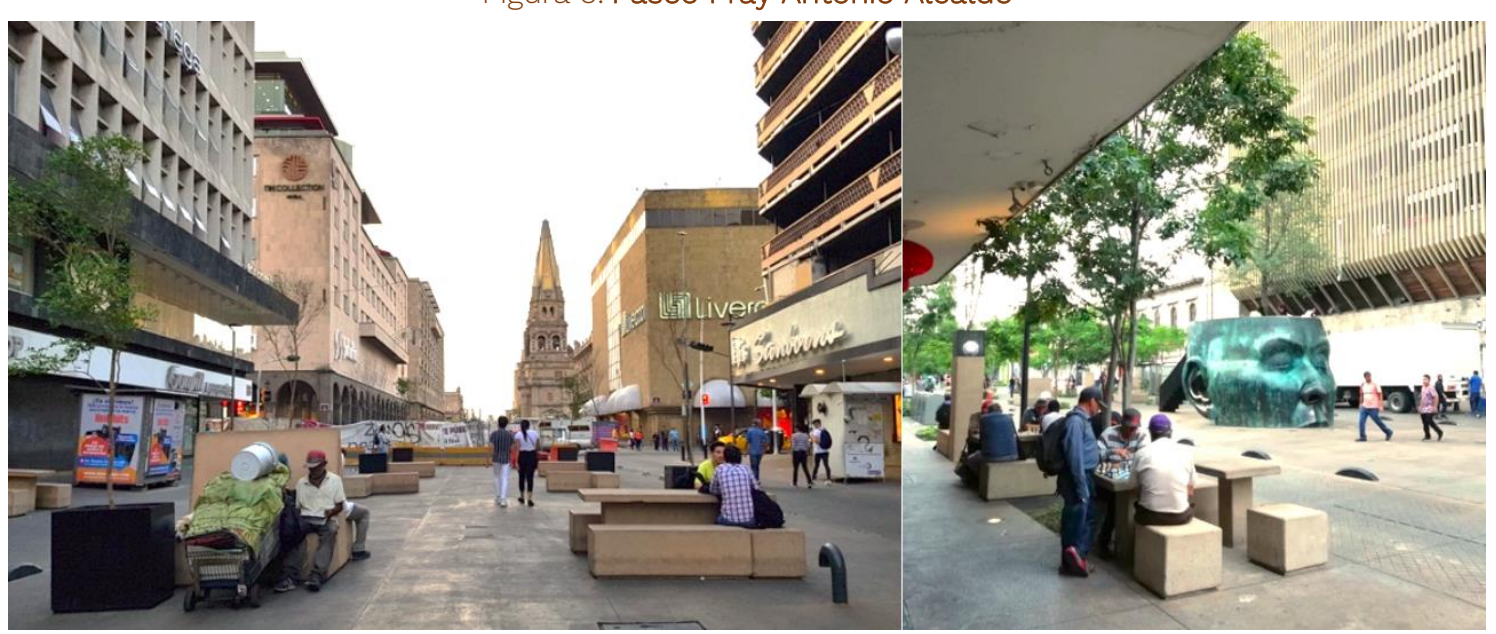

Fuente. Mayan Salgado / Carlos Crespo

ACE, 16 (47) CC BY-ND 3.0 ES | UPC Barcelona, España | Espacio público y transporte masivo: usos y percepciones. Paseo Alcalde y línea 3 SITEUR en el Área Metropolitana de Guadalajara, México. DOI: http://dx.doi.org/10.5821/ace.16.47.9631 


\section{Análisis de usos del espacio público integrado al proyecto del transporte masivo mediante encuesta de percepción}

Desde que el proyecto del Paseo Fray Antonio Alcalde fue anunciado en el año 2016, diversos sectores sociales han emitido opiniones respecto al objetivo del proyecto y sobre las posibles transformaciones que habrán de definir el futuro del contexto. Académicos, comerciantes de la zona y concesionarios del transporte público, fueron algunos de los grupos que han emitido su opinión al respecto.

Desde el núcleo académico las inquietudes se sustentaron desde el inicio en la falta de información sobre el proyecto, la difusión de este fue escasa y se reducía a las imágenes que algunos de los principales medios impresos de comunicación locales emitían, esta falta de transparencia, aunada a las múltiples inconformidades relacionadas con los cambios de uso de suelo propuestos para la zona dentro los planes parciales que acompañaban al Plan de Desarrollo Urbano de Centro de Población del municipio de Guadalajara, además de otros antecedentes como lo ocurrido en barrios tradicionales centrales como "El Retiro" y su transformación en sede de la Ciudad Creativa Digital, y sobre todo, la inexistencia de un plan de manejo para la zona del nuevo Paseo, llevaban a especular con el verdadero objetivo de este proyecto de transformación y rescate de la zona, conceptos como “limpieza social”, "despojo” y "gentrificación” se hicieron y siguen haciéndose presentes.

Por su parte, los comerciantes instalados en Av. Alcalde mostraron preocupación desde su anuncio del proyecto debido a que los cierres viales por obras no permitirían el comerciar sus productos, esta preocupación se convirtió en realidad, en febrero del año 2019, la Cámara de Comercio de Guadalajara comunicó cifras correspondientes a las afectaciones al sector siendo estas alarmantes. Desde el inicio de las obras de construcción de la línea 3 del (SITEUR) y la construcción del Paseo Fray Antonio Alcalde, solo en el tramo que comprende el Paseo, dieron como resultado 1,120 negocios con afectaciones económicas, 216 comercios tuvieron que cerrar sus puertas, representando esto una pérdida de 2 mil 700 empleos y de 590 millones de pesos en pérdidas por ventas no realizadas (El informador, 2019). Durante el proceso de construcción del proyecto del Paseo y la línea 3 del (SITEUR), la afectación a la actividad económica que se reflejó en la bancarrota de negocios que no soportaron el dilatado lapso de las obras, a la par que se abrió la puerta a la especulación inmobiliaria en virtud de que la zona aumentó su plusvalía en un $180 \%$ en los últimos 10 años y un 40\% en los últimos 5 años. Es claro apuntar que dichos proyectos y obras serán objeto de múltiples análisis en un futuro por su magnitud y trascendencia.

Los concesionarios del transporte público, por su parte, alzaron la voz respecto a su inconformidad por el desvío de 45 rutas de transporte público que transitaban por la Av. Fray Antonio Alcalde hacia vías secundarias, argumentando que esto generaría mayor congestión vial en la zona debido a que las arterias secundarias no estaban preparadas para recibir esta carga vehicular. En medio de toda esta crítica el Gobierno Municipal de la ciudad llevó a cabo un programa de rehabilitación de fachadas de valor patrimonial en todo el Paseo que incluía el retiro de pintura en mal estado, enjarres, perfilados y aplicación pintura nueva, así como un proyecto de iluminación de banquetas (Gobierno de Guadalajara, 2020).

En medio de estos argumentos que ponían y ponen en duda el éxito del Paseo, también existe una gran cantidad de voces que emiten apoyo al proyecto y lo difunden como un gran logro para la ciudad, vaticinando éxito total. Los argumentos son claros, el ganar espacio público peatonal, recreativo y de ocio a lo que anteriormente era un río de automotores circulando, se debe considerar un gran logro para la ciudad, el fomentar espacios destinados para el encuentro y, por tanto, a la cohesión social.

ACE, 16 (4.7) CC BY-ND 3.0 ES | UPC Barcelona, España | Espacio público y transporte masivo: usos y percepciones.

Paseo Alcalde y línea 3 SITEUR en el Área Metropolitana de Guadalajara, México. DOI: http://dx.doi.org/10.5821/ace.16.47.9631 
Argumentos como el anterior, son un objetivo que forma parte de todas las agendas urbanas a nivel mundial, por tanto, aunque se reconozca que los procesos para la conformación de este proyecto no fueron los más adecuados y que por ello se tenga una deuda con los afectados, el objetivo del proyecto, para estas voces, debe de ser respaldado.

En medio de visiones encontradas, desde el anuncio del proyecto hasta la fecha, la percepción de dos de los sectores mayormente implicados en una zona como el Centro Histórico de Guadalajara, sus habitantes y sus visitantes, se ha traducido en acciones concretas en el espacio, mas no se ha tenido registro alguno de ello, que es precisamente este, el cometido del presente abordaje investigativo.

A fin de conocer el proceso de entendimiento de los nuevos órdenes establecidos es que se realiza este primer ejercicio de evaluación perceptual del proyecto por parte de sus visitantes, para, en una segunda etapa, trabajar directamente con los habitantes de los perímetros circundantes al proyecto.

En esta primera etapa la encuesta de percepción se aplicó a visitantes del Paseo Fray Antonio Alcalde, cubriendo una longitud de aproximadamente 500 metros, definidos y acotados por el trayecto de paseo comprendido entre la Avenida Juárez y la Calle Reforma, teniendo al centro del tramo a la estación Catedral, la zona de estudio se seleccionó debido a la alta afluencia de visitantes. Catedral es la décima estación (tercera subterránea) partiendo desde el extremo norte de la línea y es la que se encuentra ubicada en la Cruz de Plazas. Está dispuesta bajo la Avenida 16 de septiembre entre la calle de Morelos y Juárez pasando por Calle Pedro Moreno (Figura 4).

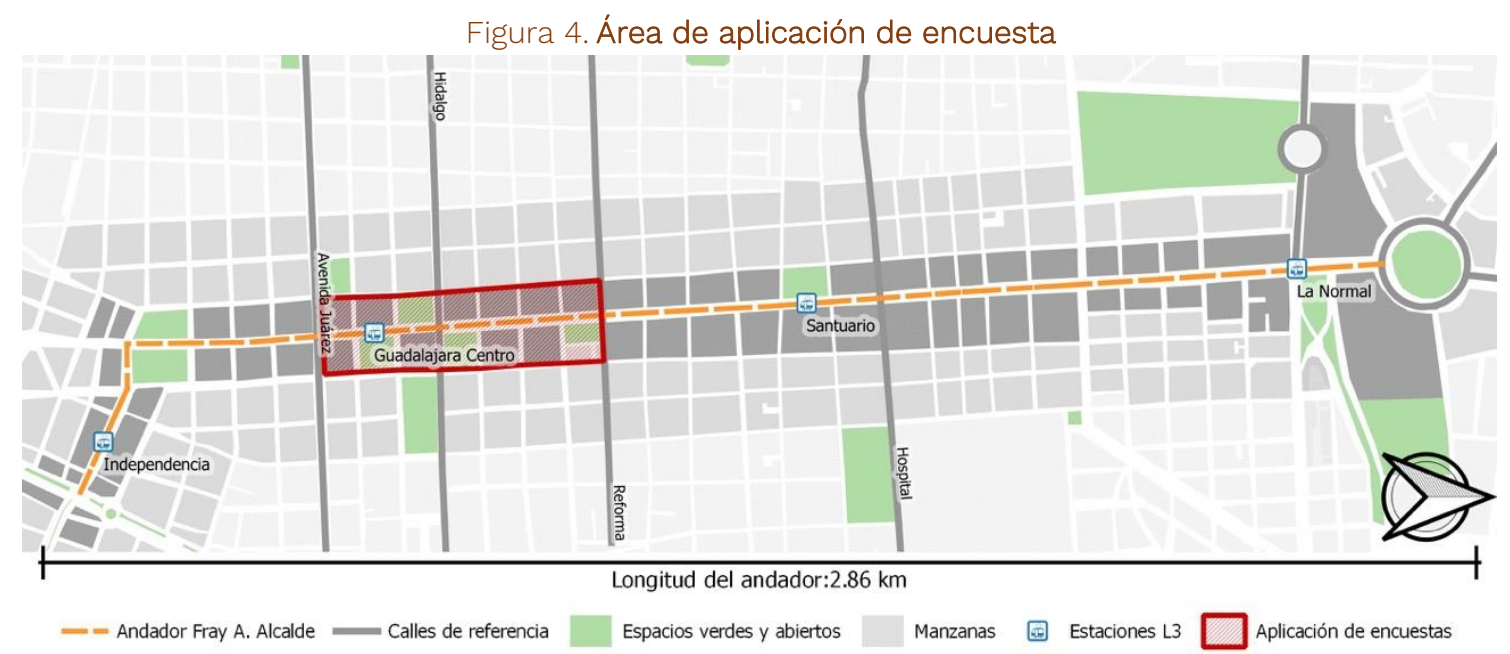

Fuente. Elaboración por parte de colaborador Yoav Alejandro Jiménez Aguas

La encuesta se aplicó a un total de 300 visitantes elegidos de forma aleatoria, el sujeto de encuesta solo tenía una condicionante que debía cumplir, ser mayor de 15 años. Su aplicación se llevó a cabo durante el mes de julio del 2019. La batería de preguntas se conformó por 4 secciones: Motivo y frecuencia de visita; conocimiento del proyecto; usos socio-espaciales; y valoración del contexto espacial (detalles en Tabla 1).

ACE, 16 (4.7) CC BY-ND 3.0 ES | UPC Barcelona, España | Espacio público y transporte masivo: usos y percepciones. Paseo Alcalde y línea 3 SITEUR en el Área Metropolitana de Guadalajara, México. DOI: http://dx.doi.org/10.5821/ace.16.47.9631 
Tabla 1. Criterios de la Encuesta de percepción - variables, indicadores y resultados

\begin{tabular}{|c|c|c|c|}
\hline \multicolumn{4}{|c|}{ Muestra 300 visitantes /perfil mayor de 15 años } \\
\hline Variable & Indicador & Resultados & $\%$ \\
\hline \multirow{2}{*}{$\begin{array}{l}\text { Motivo y } \\
\text { frecuencia de } \\
\text { visita }\end{array}$} & Motivo & $\begin{array}{l}\text { Motivos de tránsito } \\
\text { y recreativos }\end{array}$ & 74 \\
\hline & Frecuencia & $\begin{array}{l}1 \text { a } 5 \text { veces por } \\
\text { mes }\end{array}$ & 80 \\
\hline \multirow{2}{*}{$\begin{array}{l}\text { Conocimiento } \\
\text { del proyecto }\end{array}$} & Conoce el proyecto & parcialmente & 31 \\
\hline & Identifica la extensión & parcialmente & 45 \\
\hline \multirow[t]{5}{*}{$\begin{array}{l}\text { Usos socio- } \\
\text { espaciales }\end{array}$} & $\begin{array}{l}\text { Usos predominantes en el nuevo espacio } \\
\text { público }\end{array}$ & Recreativo & 72 \\
\hline & Grado de utilización del Paseo & Moderado & 57 \\
\hline & $\begin{array}{l}\text { Nivel de conocimiento en relación con las } \\
\text { actividades promovidas por el municipio } \\
\text { para la activación del nuevo proyecto }\end{array}$ & Redes sociales & 28 \\
\hline & $\begin{array}{l}\text { Grado de compatibilidad entre la } \\
\text { configuración arquitectónica del espacio y } \\
\text { los usos o actividades que se realizan; }\end{array}$ & Congruente & 76 \\
\hline & $\begin{array}{l}\text { Los usos o actividades que les gustarían } \\
\text { realizar en el espacio y por motivos de } \\
\text { configuración arquitectónica ven } \\
\text { complicado llevar a cabo }\end{array}$ & $\begin{array}{l}\text { Eventos ferias, } \\
\text { desfiles, conciertos } \\
\text { y promoción del } \\
\text { arte }\end{array}$ & 83 \\
\hline \multirow{2}{*}{$\begin{array}{l}\text { Valoración del } \\
\text { contexto } \\
\text { espacial }\end{array}$} & Actividades sociales y culturales & Satisfactorio & 81 \\
\hline & Seguridad en la zona & seguridad & 70 \\
\hline
\end{tabular}

Fuente: Investigación de campo (encuesta 2019). Elaboración propia

A continuación, se muestran algunos de los resultados obtenidos.

\subsection{Motivos y frecuencia de visita.}

Esta sección de la encuesta tiene por objetivo conocer las motivaciones y la frecuencia de visita al Paseo, a unos meses de inaugurado y aún sin entrar en función ninguna de las tres estaciones de la línea 3 del (SITEUR) que aloja el Paseo, con la finalidad de conocer el impacto a corto plazo del proyecto en la dinámica social de la zona y, sobre todo, sentar la bases para generar reflexiones futuras relacionadas con el impacto que tiene sobre los motivos y la frecuencia de uso espacial una vez que entrará en función la nueva línea.

Del total de los encuestados, el $41 \%$ van de paso, 33\% acuden por motivos recreativos, $18 \%$ de compras y $8 \%$ por motivos laborales. Respecto a la frecuencia de visita, el $44 \%$ de los encuestados manifestó visitar una sola vez al mes dicho espacio, mientras que un 36\% asiste de 2 a 5 veces al mes a este espacio. Con relación a los días específicos de visita, los encuestados manifestaron en su mayoría visitar el espacio durante los fines de semana 48\% y, predominantemente por la tarde, así lo contestó el $72 \%$ de los encuestados.

\subsection{Conocimiento del proyecto}

Como se hizo mención el proyecto "Paseo Fray Antonio Alcalde" tiene una longitud total de $2.5 \mathrm{~km}$, abarcando un eje que parte, al norte, de la Glorieta de la Normal y llega, al sur, hasta la zona conocida como los dos templos (San Francisco y Aranzazú). Sin embargo, existe dentro del total del andador zonas específicas que acumulan la mayor actividad comercial y turística, puntos turísticos como la Catedral de Guadalajara, la Rotonda de los Jaliscienses Ilustres, Plaza de Armas, Plaza Guadalajara, el Palacio de Gobierno y múltiples espacios comerciales como los es la Plaza de la Tecnología, entre otros puntos de atracción, configuran esta zona que comprende apenas el 28\% de la extensión longitudinal del proyecto (700 metros). El proyecto también tiene por objetivo descentralizar la

ACE, 16 (4.7) CC BY-ND 3.0 ES | UPC Barcelona, España | Espacio público y transporte masivo: usos y percepciones. 12 Paseo Alcalde y línea 3 SITEUR en el Área Metropolitana de Guadalajara, México. DOI http://dx.doi.org/10.5821/ace.16.47.9631 
actividad comercial y turística, ampliándose durante todo el recorrido peatonal proyectado, teniendo en consideración este aspecto se cuestionó a los encuestados el grado de conocimiento respecto a la extensión total del proyecto, a esto, solo el 31\% de los encuestados manifestó conocer el proyecto, y de ellos, solo el 45\% definió correctamente la extensión de este.

\subsection{Usos socio espaciales}

En este apartado se buscó conocer la percepción del visitante con respecto a: usos predominantes en el nuevo espacio público; grado de utilización del Paseo; nivel de conocimiento en relación con las actividades promovidas por el municipio para la activación del nuevo proyecto; grado de compatibilidad entre la configuración arquitectónica del espacio y los usos o actividades que se realizan; y los usos o actividades que les gustarían realizar en el espacio y por motivos de configuración arquitectónica ven complicado llevar a cabo.

El uso socio espacial predominante según la percepción de sus visitantes es recreativo, seguido de un uso de tránsito, esto es lógico, puesto que se habla de un espacio público que funge como contenedor de entornos diseñados para la permanencia y realización de actividades estáticas que se distribuyen linealmente sobre una superficie que funciona como perfecto medio de conexión entre la zona norte y la zona sur del Centro Histórico. En un tercer orden, los visitantes perciben que el espacio tiene una vocación comercial, por lo que colocan a esta actividad económica como un aspecto secundario cuando se trata de disfrutar del proyecto. Esta lógica concuerda con los objetivos del proyecto.

Respecto al grado de utilización del Paseo, la mayoría de los visitantes consideran que su uso es aún moderado (57\%), esto puede deberse a dos factores principalmente: el primero tiene que ver directamente con el hecho de que la línea 3 del (SITEUR) aún no estaba en funcionamiento, como se planteó en el anterior apartado, el Paseo ayudará a generar una conexión peatonal o por medios no motorizados con las 3 estaciones que se encuentran contiguas al mismo; el segundo factor, sobre el que se debe poner especial atención, es la centralización de las actividades del Paseo sobre el área de Catedral, 500 metros de longitud norte-sur, tramo en el que se concentran la mayoría de los atractivos para los visitantes.

El municipio de Guadalajara, siendo consciente de la necesidad de promover el trayecto completo del Paseo, en la búsqueda de descentralizar las actividades, han realizado eventos culturales y recreativos en diferentes tramos de los $2.5 \mathrm{~km}$ de paseo (recorridos históricos, talleres para niños, música y baile), estos eventos se llevan a cabo frecuentemente durante la semana y fines de semana, y su medio de difusión es principalmente redes sociales. Al respecto se cuestionó a los visitantes sobre el conocimiento de dichas actividades a lo que solo el $28 \%$ manifestó conocer dichas actividades.

Sobre el grado de compatibilidad entre la configuración arquitectónica y los usos y actividades desarrolladas en este espacio, los visitantes encuestados manifestaron en su mayoría (76\%) que existe congruencia ya que las actividades desarrolladas, en su mayoría, son recreativas. El resto de los visitantes manifestó encontrar incongruencia en sus usos en relación directa con el comercio ambulante que aún se encuentra presente en ciertos tramos del Paseo. Posterior a conocer la apreciación sobre compatibilidad diseño - uso, se cuestionó sobre las actividades que les gustaría realizar y que por motivos de diseño no pueden llevar a cabo en el espacio, con el fin de evaluar no solo lo existente sino también lo que las personas desearían que el espacio propiciara, a lo que la mayoría fue participativa en sugerir nuevos usos para el espacio, entre ellos: la realización de eventos como desfiles, conciertos y ferias (53\%); la promoción del arte en el espacio (30\%); y la realización de algunos deportes (17\%).

ACE, 16 (47) CC BY-ND 3.0 ES | UPC Barcelona, España | Espacio público y transporte masivo: usos y percepciones.

Paseo Alcalde y línea 3 SITEUR en el Área Metropolitana de Guadalajara, México. DOI http://dx.doi.org/10.5821/ace.16.47.9631 
Es importante detenerse un momento para reflexionar particularmente sobre las primeras dos propuestas. El deseo relacionado con que el espacio contenga eventos como los desfiles tiene relación directa con la vocación pasada del lugar, ya que, sobre la Av. Alcalde, tenían lugar todos los desfiles conmemorativos de la ciudad (desfile militar en conmemoración a la Independencia Nacional, desfile conmemorativo de la Revolución Mexicana, desfile conmemorativo del día del trabajo, desfile del Encuentro Internacional del Mariachi y la Charrería, entre otros), tras el inicio de las obras del tren, los desfiles cambiaron de sede para desarrollarse sobre avenida Chapultepec, espacio poco propicio para su desarrollo debido a sus características espaciales y, sobre todo, debido a ser etiquetado históricamente como uno de los espacios de uso comercial y recreativo de las clases medias - altas de la ciudad. Es evidente el deseo de devolver al centro de la ciudad esta capacidad festiva. Por otro lado, el anhelo de integrar el arte a este espacio tiene que ver con el impulso actual que está dando el gobierno en turno al desarrollo de piezas gráficas y escultóricas dentro de la Zona Metropolitana de Guadalajara, impulso que aún no logra verse reflejado en la zona centro, puesto que, del total de piezas artísticas expuestas en la ciudad y para la ciudad, un limitado porcentaje se encuentra en el área central de la ciudad, a pesar de que en esta zona se encuentra ubicada la sede de Artes, el Ex Claustro de Santa María de Gracia, perteneciente al Centro Universitario de Arte, Arquitectura y Diseño de la Universidad de Guadalajara; son otras las zonas de la ciudad que se benefician de ello. El Paseo solo cuenta con un solo elemento escultórico dentro de todo su trayecto, (no incluido en el proyecto original), Árbol adentro del artista José Fors, el cual se ha vuelto un polo de atracción para locales y visitantes. Sobre este anhelo de espacios para el arte, también se puede subrayar que en el diseño del espacio no se contempló la creación de anfiteatros, o explanadas dispuestas para la representación de las artes escénicas o la danza. Las demandas tienen claro fundamento.

\subsection{Valoración del paseo}

A fin de conocer la valoración general del Paseo, se sometieron a evaluación las características físicas del espacio, la capacidad de éste para llevar a cabo actividades sociales y culturales, y la percepción de seguridad.

Las características físicas del espacio fueron valoradas como muy satisfactorias por el $81 \%$ de los encuestados. Los elementos de la configuración espacial mejor valorados por los visitantes de la zona son: las condiciones e imagen de los edificios circundantes al Paseo y las mejoras respecto a la accesibilidad universal. En un segundo orden de valoración, clasificado como una mejora regular, se encuentran la iluminación del paseo, el nuevo mobiliario urbano colocado y el aumento de masa vegetal. La calificación negativa respecto al diseño del espacio se les dio a los espacios para las actividades infantiles, debido a la carencia total de juegos infantiles a lo largo de los $2.5 \mathrm{~km}$ de paseo, elemento sumamente valorado por las familias que visitan el centro y que no fue contemplado en el diseño.

En lo que respecta a la capacidad para llevar a cabo actividades sociales y culturales el espacio también fue valorado como muy satisfactorio por los encuestados. Las actividades con mayor factibilidad para desarrollarse fueron: caminar y platicar, esto se debe fundamentalmente a que el proyecto pone al peatón como prioridad. En un segundo orden de factibilidad, clasificada como regular, se encuentra la capacidad de consumir productos alimenticios en el espacio, esto se debe principalmente a que solo se cuenta con bancas en la zona y su dimensión es reducida, solo un pequeño tramo del recorrido cuenta con bancas y mesas. Las actividades que fueron catalogadas como poco factibles de llevarse a cabo fueron las artísticas y las infantiles, el juego. El Paseo, como ya se mencionó, no cuenta con espacios diseñados para el desarrollo de actividades artísticas y mucho menos para el juego.

ACE, 16 (47) CC BY-ND 3.0 ES | UPC Barcelona, España | Espacio público y transporte masivo: usos y percepciones.

Paseo Alcalde y línea 3 SITEUR en el Área Metropolitana de Guadalajara, México. DOI: http://dx.doi.org/10.5821/ace.16.47.9631 
El último elemento sometido a evaluación fue la seguridad en la zona, los resultados arrojan que la mayoría de los encuestados manifestaron sentirse regularmente seguros en el espacio (70\%). Es prematuro conocer si el desarrollo del proyecto ha retribuido positivamente a aminorar la percepción de inseguridad en la zona, se requerirá de más tiempo para ello, se considera que la apreciación actual tiene que ver con el pasado conflictivo del espacio.

Basados en los resultados expuestos en este apartado es pertinente generar algunas reflexiones que emergen del ejercicio de encuesta y de la observación de los investigadores en campo, mismas que se encuentran vinculadas con el estado presente del proyecto, pero también sobre algunos fenómenos sociales que se comienzan a vislumbrar y representan retos con miras a futuro, un futuro en el que el uso del espacio público se complementa con actividades relacionadas con la movilidad motorizada y no motorizada.

\section{Reflexiones. Retos pendientes para lograr la vinculación entre la Línea 3 del SITEUR y el Paseo Fray Antonio Alcalde}

Tras el abordaje en campo, se hizo evidente que el Paseo representa para sus visitantes un cambio positivo, un proyecto que mejora sustancialmente la dinámica del centro histórico, sin embargo, debido al poco tiempo de inauguración y apertura de esta obra, resultó imposible aislar de los entrevistados sus recuerdos inmediatos con relación a lo que era anteriormente la Avenida Alcalde, esto fue evidenciado a través de las constantes comparativas entre lo nuevo y lo viejo, es decir, la evaluación del espacio sustentada en el contraste con los recuerdos aún vívidos de lo representaba para ellos esta avenida: Tráfico, estrés, inseguridad, accidentes, suciedad, oscuridad, descuido y caos.

El proyecto se visualiza como en territorio ganado y se evalúa según las oportunidades que representa para lograr mejorar las condiciones de un Centro Histórico que por años había permanecido en abandono, voluntario o involuntario, por parte de las autoridades. No obstante, el proyecto enfrenta algunos retos importantes.

El primer gran reto que debe afrontar el actual Paseo Fray Antonio Alcalde es la generación de un plan de manejo integral para la zona, instrumento jurídicamente reconocido y herramienta normativa fundamental para la gestión, la vinculación y organización entre todos los actores implicados, que contenga planes y proyectos estratégicos para garantizar que las transformaciones, sociales, culturales, económicas y ambientales, derivadas de esta importante obra, no trastornan los diferentes usos coexistentes en la zona, fomentando así una efectiva vinculación entre criterios de movilidad, habitabilidad, identidad, patrimonio, activación económica y actividades recreativas y culturales. Un plan que garantice el mejoramiento en las condiciones de vida en la zona.

El segundo reto con el que se topa este espacio público es la descentralización de las actividades. El Paseo, en su extensión completa de $2.5 \mathrm{~km}$, alberga fronteras intangibles que requieren ser disueltas, la centralización de actividades en torno al tramo comprendido entre la Catedral de Guadalajara y el Jardín San Francisco (dos templos), polos de atracción del turismo nacional e internacional, es evidente y representa un reto de enorme importancia, este tramo representa aproximadamente el 30\% de la longitud total del Paseo, 800 metros lineales, la centralización de las actividades recreativas y culturales hacen que el resto del Paseo luzca la mayoría del tiempo desolado. Considerando al Paseo como un conector peatonal entre las 3 estaciones del TEU es importante que la diversidad de usos se consolide a lo largo de todo el trayecto, evitando así fronteras y tramos que representan espacios intersticiales que inviten poco al desarrollo de actividades de flujo y permanencia.

ACE, 16 (47) CC BY-ND 3.0 ES | UPC Barcelona, España | Espacio público y transporte masivo: usos y percepciones.

Paseo Alcalde y línea 3 SITEUR en el Área Metropolitana de Guadalajara, México. DOI: http://dx.doi.org/10.5821/ace.16.47.9631 
El tercer reto importante es garantizar un espacio público que promueva a partir de su diseño, diversidad de usos y actividades sociales, correspondientes a la heterogeneidad social que caracteriza a la vida urbana. El Paseo Fray Antonio Alcalde actualmente representa un soporte material homogéneo, $2.5 \mathrm{~km}$ de patrones de diseño repetitivos sustentan la obra. En esta plataforma, o "lienzo en blanco" como lo llaman los arquitectos de la obra, las posibilidades recreativas emergentes son escasas y se reducen al acto de caminar de un punto a otro sin que existan en el trayecto elementos que evoquen actividades diversas por parte de quienes transitan por él, dicho esto, un gran reto con el que se topará el proyecto será la posible gran dependencia por parte de sus usuarios sobre la organización de eventos programados y gestionados por los administradores del mismo o, en su caso, por el comercio que se establezca a lo largo del paseo. Ante este contexto el visitante ávido de explotar el uso recreativo del espacio se encuentra supeditado a un contenido programado. Partiendo de que el espacio público debe ser una plataforma abierta a la coproducción social de sus contenidos, y por tanto, en constante proceso de estructuración social, no viene bien condicionar la acción social a un guion prediseñado y fácilmente manipulable. Los espacios públicos deben concebirse como plataformas en constante proceso de estructuración por parte de quienes los usan, los viven, los habitan, es por ello que estos deben garantizar su flexibilidad y adaptabilidad ante el accionar cotidiano, ser receptores y precursores de la heterogeneidad que representa a la urbano.

Estos son los tres principales retos detectados a partir del cruce de información derivada de las encuestas realizadas y la observación en sitio, retos que deberán atenderse para garantizar el logro del objetivo planteado, lograr dentro del primer cuadro del Centro Histórico de Guadalajara el establecimiento de la intermodalidad como forma de desplazamiento y su vinculación con espacios públicos abiertos, accesibles y que fomenten del intercambio social.

\section{Conclusiones}

La consideración del estudio de un megaproyecto en la Zona Metropolitana de Guadalajara como lo es la línea 3 SITEUR es una oportunidad para una investigación de carácter multidisciplinar que abonará a un sentido crítico sobre de los impactos que conlleva este tipo de intervención. Es claro que la puesta en marcha de un proyecto de infraestructura en movilidad y transporte masivo, un proyecto de futuro a mediano y largo plazo, trae como consecuencia una serie de impactos territoriales, sociales, económicos y políticos. Por tanto, la preocupación de los efectos y consecuencias de carácter socio espacial son objeto de este artículo, en virtud de las transformaciones que se han realizado producto de la reestructuración urbana

Las infraestructuras destinadas a la movilidad y los espacios públicos en la ciudad deben pensarse, proyectarse y concebirse en conjunto, vinculados estrechamente, para generar conexiones en red que permitan la intermodalidad, habiliten dinámicas de proximidad urbana, el cambio en las escalas vivenciales del ser urbano y, con ello, posibiliten la topofilia, el reencuentro afectivo con los lugares y con la diversidad de estímulos que emergen de la heterogeneidad social que los distingue.

Hoy en día nuestras ciudades se encuentran en una etapa de redefinición de los patrones funcionales, de sus escalas, de sus densidades, de sus ritmos y de las formas de interacción humana, las medidas de confinamiento y distanciamiento social han puesto sobre la mesa la oportunidad de afrontar esta nueva etapa venidera apropiándose de la ciudad fortaleciendo prácticas fundamentales para el ser humano como la recreación y el ocio. La disposición de una mayor superficie espacial es fundamental, por ello, el Paseo Fray Antonio Alcalde representa una obra dispuesta, habilitada para estos fines, un ejercicio de transformación espacial que merece afrontar sus retos y garantizar mixtura en sus usos, apertura a las diversas formas de apropiación social, convirtiéndose en plataforma de diálogo constante entre los diversos agentes implicados en dotar de sentido al acontecimiento, garantizar su facultad política.

ACE, 16 (47) CC BY-ND 3.0 ES | UPC Barcelona, España | Espacio público y transporte masivo: usos y percepciones.

Paseo Alcalde y línea 3 SITEUR en el Área Metropolitana de Guadalajara, México. DOI. http://dx.doi.org/10.5821/ace.16.47.9631 
Es evidente que este abordaje investigativo que desarrolla un análisis de la percepción y los usos sociales de la ciudad en un proyecto de gran envergadura en la ciudad de Guadalajara, como es el Paseo Fray Antonio Alcalde, se vio interrumpido por los cambios que han tenido que afrontar la ciudad debido a la pandemia Covid-19, desde marzo del 2020 y que ha hecho que el discurso y las acciones del gobierno municipal, estatal y nacional para frenar la pandemia, hayan hecho retroceder la vida política y comunitaria del país; la propuesta de quédate en casa ha hecho desaparecer del discurso público a la ciudad y a los ciudadanos, y se ha aprovechado para hacer una estigmatización de los espacios públicos congestionados, el peligro del espacio público está allí, en un momento de crisis sanitaria, económica, financiera, social, política, urbana y ecológica.

El estudio generado sobre la vinculación entre la Línea 3 del SITEUR (infraestructura de movilidad) y el Paseo Fray Antonio Alcalde (espacio público de escala metropolitana) ha tenido como fin evaluar la potencialidad implícita en el vínculo estrecho entre prácticas de flujo y prácticas de permanencia para lograr entornos urbanos dispuestos a la mejora en la calidad de vida de los habitantes, permanentes u ocasionales. El paso subsecuente en la evaluación representa un cambio de escala, hoy en día este vínculo requiere verse reflejado y potencializado a escala barrial, ahí donde la vida del ser urbano se desarrolla cotidianamente y que, bajo las nuevas condicionantes de vida, refuerza su sentido.

\section{Agradecimientos}

Los autores de este articulo agradecemos el apoyo de la Universidad de Guadalajara, en especial el recibido por el Instituto de Investigación y Estudios de la Ciudad. Además damos un especial agradecimiento al trabajo realizado por Pablo Chavarría, José Luis Vite y Mayan Salgado, su colaboración durante el proceso de levantamiento de información en campo fue de suma importancia para la investigación. A Yoav Alejandro Jiménez por su apoyo en la elaboración de los gráficos mostrados en este trabajo. Su colaboración tiene un especial valor para el desarrollo de este trabajo de investigación.

\section{Autoría}

El primer autor ha fundamentado los antecedentes y el contexto histórico de la ciudad y del espacio público, el marco teórico del espacio público, antecedentes y contexto histórico del Proyecto del Paseo Alcalde de la línea 3 del SITEUR, participación en el diseño general de criterios de la encuesta en el grupo de trabajo y coordinación del trabajo de campo. Conclusiones, impacto del proyecto, escenarios futuros. El segundo autor ha fundamentado el marco teórico de la percepción y usos en la ciudad contemporánea, el marco teórico del espacio público. Realización de análisis de uso del espacio público a partir de la percepción. Análisis de datos y resultados. Conclusiones, impacto del proyecto, escenarios futuros. Obtención de datos correspondientes a los antecedentes y entorno del proyecto del paseo Alcalde. Participación en el diseño general de criterios de la encuesta en el grupo de trabajo y coordinación del trabajo de campo. El tercer autor ha realizado la contextualización y marco teórico de los megaproyectos, las infraestructuras de movilidad y de transporte masivo. Contextualización y antecedentes de la línea 3 del SITEUR en Guadalajara. Complemento de datos en los antecedentes y entorno del proyecto del paseo Alcalde. Participación en el diseño general de criterios de la encuesta en el grupo de trabajo. Conclusiones de la importancia de los megaproyectos.

Conflicto de intereses: Los autores declaran que no hay conflicto de intereses.

ACE, 16 (47) CC BY-ND 3.0 ES | UPC Barcelona, España | Espacio público y transporte masivo: usos y percepciones.

Paseo Alcalde y línea 3 SITEUR en el Área Metropolitana de Guadalajara, México. DOI http://dx.doi.org/10.5821/ace.16.47.9631 


\section{Bibliografía}

Arellano Ríos, A. (2018). El transporte público en el área Metropolitana de Guadalajara: agenda, proyectos y "gatopardismo". Revista Mexicana de Análisis Político y administración Pública, VII(1), 1132.

Calonge Reillo, F. (2017). Infraestructura de transporte masivo y sustentabilidad urbana. En F. Calonge Reillo (Ed.), La sustentabilidad urbana vía muerta. Gobernanza y participación ciudadana ante la construcción de infraestructuras de transporte masivo. (pp. 9-46). Argentina: Estudios Sociológicos Editora.

Calonge Reillo, F. (2017). ¿Qué factores aumentan la aprobación al gobierno en un proyecto de infraestructura urbana? La construcción de la Línea 3 del Tren Ligero en el área Metropolitana de Guadalajara, México. GAAP Nueva época, (21), 109-124. DOI: https://doi.org/10.24965/gapp.v0i21.10537

Cámara Nacional de Comercio. (21 de febrero de 2019). Se han perdido cuatro de cada 10 empleos en zona de Línea 3. El informador, 21-24.

Carrillo Regalado, S. (1994). La Carrillo Regalado, S. (1994). La problemática del transporte público de pasajeros en el Área metropolitana de Guadalajara. Carta económica regional (9), 1-6. DOI: https://doi.org/10.32870/cer.v0i9.7174

Córdova Espña, M. (2010). La movilidad urbana y el transporte: en búsqueda de un nuevo paradigma. En V. González Romero, dos décadas de desarrollo en Jalisco (pp. 161-180). Guadalajara: Secretaría de Planeación. Gobierno de Jalisco.

Díaz Orueta, F. (2009): El impacto de los megaproyectos en las ciudades españolas. Hacia una agenda de investigación. Estudios Demográficos y Urbanos, 24(1) 193-218. DOI: https://doi.org/10.24201/edu.v24i1.1348

Díaz Orueta, F. (2015). Megaproyectos urbanos y modelo de ciudad: el ejemplo de Madrid Río. Cuaderno Urbano, 19(19): 179-200. DOI: https://doi.org/10.30972/crn.1919813

Downs, R. M. (1970). Geographic space perception. Past upproahes and future prospects. Progress in Geography. International Review of current Research, 2, 65-108.

Gobierno del Estado de Jalisco. (2018). Plan Estatal de Gobernanza y Desarrollo de Jalisco 2018-2024. Visión 2030. Recuperado de https://plan.jalisco.gob.mx/sites/default/files/2019-06/Plan-Estatal-deGobernanza-y-Desarrollo-de-Jalisco v2.pdf

Gobierno de Guadalajara. (2020). Guadalajara inicia la rehabilitacoón de fachadas de fincas del Paseo Alcalde. Recuperado de https://guadalajara.gob.mx/comunicados/guadalajara-inicia-rehabilitacionfachadas-fincas-del-paseo-alcalde

Gobierno de Guadalajara (2017) Plan de Desarrollo Urbano de Centro de Población Visión Quinto Centenario 2017-2042. Recuperado de https://paseoalcalde.guadalajara.gob.mx

Gobierno de Guadalajara. (2017). Plan Maestro Centro de Guadalajara. Recuperado de https://paseoalcalde.guadalajara.gob.mx

ACE, 16 (47) CC BY-ND 3.0 ES | UPC Barcelona, España | Espacio público y transporte masivo: usos y percepciones. Paseo Alcalde y línea 3 SITEUR en el Área Metropolitana de Guadalajara, México. DOI http://dx.doi.org/10.5821/ace.16.47.9631 
Gobierno de Guadalajara. (2017). Plan Parcial de Desarrollo Urbano. Distrito Urbano 1 "Centro Metropolitano". Subdistrito Urbano 05 "Centro Histórico". Recuperado de https://paseoalcalde.guadalajara.gob.mx

Gobierno de Guadalajara. (2016). Plan rector del Polígono de Intervención Urbana Especial (PIUE.) Corredor Alcalde - Centro Histórico Recuperado de https://paseoalcalde.guadalajara.gob.mx

Instituto de Información Territorial. (2013). El ruido en Guadalajara como fuente de contaminación ambiental y su efecto en la salud humana. Guadalajara. Recuperado de https://iieg.gob.mx/contenido/GeografiaMedioAmbiente/iterritorial notatecnica 08 2013.pdf

Miralles-Guasch, C. y Cebollada i Frontera, A. (2003). Movilidad y transporte. Opciones políticas para la ciudad. Barcelona, España: Laboratorio de Alternativas.

Lefebvre, H. (1974). The production of space. Londres: Blackwell.

Park, R. E. (1999). La ciudad y otros ensayos de ecología urbana. Barcelona, España Editoria del Serval.

Pérez López, R. (2019) Producción de la ciudad en tiempos de globalización. Impactos socioterritoriales de los megaproyectos. Bitácora Urbano Territorial, 29(1), 13-22. DOI: http://dx.doi.org/10.15446/bitacora.v29n1.66482

Roa, N. (2010) Megaproyectos de transporte: Instituciones, política y gestión técnica de recursos. Revista de ingeniería. 32: 88-94.

Romero Pérez, F. J. (2011). Movilidad urbana en la gran Guadalajara. Trazando el rumbo. Guadalajara, Mexico: Pandora.

Suárez Pradilla, M. y Roca Cladera, J. (2019). Efecto de una infraestructura de transporte viaria en la estructura urbana: caso de estudio: autovía orbital B-40 de Barcelona. A. ACE: Architecture, City and Environment, 14(41), 131-150. DOI: http://dx.doi.org/10.5821/ace.14.41.8958

Talledos, E. e Ibarra, M. (2016) Megaproyectos en México. Una Lectura Crític. Ciudad de México, México: Facultad de Filosofía y Letras. Universidad Nacional Autónoma de México. Ítaca.

ACE, 16 (47) CC BY-ND 3.0 ES | UPC Barcelona, España | Espacio público y transporte masivo: usos y percepciones. 